\title{
Cobalt(II) Chloride-Mediated Synthesis of $\beta$-Enamino Compounds under Solvent-Free Conditions
}

\author{
Zhan-Hui Zhang ${ }^{*, a}$ and Jin-Yong $\mathrm{Hu}^{b}$ \\ ${ }^{a}$ The College of Chemistry \& Material Science, Hebei Normal University, Shijiazhuang 050016, China \\ ${ }^{b}$ Division of Science and Technology, Hebei Normal University, Shijiazhuang 050016, China
}

\begin{abstract}
Uma variedade de $\beta$-enaminonas e $\beta$-enamino ésteres foi sintetizada pela reação de compostos 1,3-dicarbonílicos com aminas na presença de quantidade catalítica de cloreto de cobalto(II), à temperatura ambiente na ausência de solventes. O procedimento experimental é simples e os produtos são isolados em altos rendimentos.
\end{abstract}

A variety of $\beta$-enaminones and $\beta$-enamino esters can be synthesized by the reaction of 1,3dicarbonyl compounds with amines in the presence of a catalytic amount of cobalt(II) chloride at room temperature under solvent-free condition. The experiment procedure is simple, and the products are straightforwardly isolated in high yields.

Keywords: $\beta$-enaminones, $\beta$-enamino esters, 1,3 -dicarbonyl compounds, amines, cobalt(II) chloride, solvent-free conditions

\section{Introduction}

$\beta$-Enaminones have attracted much attention due to the fact that they are important synthons for the synthesis of many biologically active compounds such as dopamine auto-receptor agonists, ${ }^{1}$ acetylcholinestersase inhibitors ${ }^{2}$ and anticonvulsants. ${ }^{3}$ They are also useful intermediates for the preparation of several aminoacids, ${ }^{4}$ aminols, ${ }^{5}$ peptides and alkaloids. ${ }^{6}$ Thus it is very important to search for a convenient and efficient method for the synthesis of this type of compounds. ${ }^{7}$ Several methods have been developed for their synthesis including the reactions of imidoyl chlorides with acetonyltributyltin in the presence of palladium catalyst, ${ }^{8}$ the addition of amines to acylacetylenes, ${ }^{9}$ acylation of metalated ketimines using acylbenzotriazoles as acylating agents, ${ }^{10}$ the reactions of lithiated enamines with diethyl carbonate or benzyl chloroformate, ${ }^{11}$ the addition of ester or amide enolates to nitriles, ${ }^{12}$ palladium-assisted amination of $\alpha$-keto olefins $^{9}$ or amination with $O$-methylhydroxylamines in the presence of base, ${ }^{13}$ and the reactions of alkyl azides and $\beta$-ketoesters in a one-pot procedure using $10 \% \mathrm{Pd} / \mathrm{C}$ catalyst and hydrogen. ${ }^{14}$ However, the most commonly used and straightforward approach for the preparation of

*e-mail: zhanhui@mail.nankai.edu.cn these compounds is the direct condensation of $\beta$-dicarbonyl compounds and amines, in which the azeotropic removal of water is usually required under reflux using a Dean Stark trap in aromatic solvent. ${ }^{15}$ Some improved procedures have been also reported to utilize protonic acids such as $\mathrm{HCl},{ }^{16} \mathrm{H}_{2} \mathrm{SO}_{4},{ }^{17} p$-TSA, ${ }^{18}$ ultrasound/ $\mathrm{HAc},{ }^{19}$ Lewis acids such as $\mathrm{BF}_{3} \cdot \mathrm{OEt}_{2},{ }^{20} \mathrm{Zn}\left(\mathrm{ClO}_{4}\right) \cdot 6 \mathrm{H}_{2} \mathrm{O},{ }^{21}$ $\mathrm{CeCl}_{3} \cdot 7 \mathrm{H}_{2} \mathrm{O},{ }^{22} \mathrm{NaAuCl}_{4}{ }^{23} \mathrm{Bi}(\mathrm{OTf})_{3}{ }^{24}$ and heterogeneous catalyst like natural clays, ${ }^{25}$ silica gel, ${ }^{26}$ montmorillonite $\mathrm{K} 10,{ }^{27}$ sulfated zirconia, ${ }^{28}$ silica chloride ${ }^{29}$ and microwaves/ $\mathrm{K}-10 .{ }^{30}$ Recently, $\mathrm{I}_{2},{ }^{31} \mathrm{InBr}_{3},{ }^{32} \mathrm{Zn}(\mathrm{OAc})_{2} \cdot 6 \mathrm{H}_{2} \mathrm{O},{ }^{33}$ $\left[\mathrm{EtNH}_{3}\right] \mathrm{NO}_{3},{ }^{34} \mathrm{Sc}(\mathrm{OTf})_{3}{ }^{35}$ and $\mathrm{HClO}_{4} \cdot \mathrm{SiO}_{2}{ }^{36}$ have been employed to promote this reaction. This condensation reaction has also been performed in water. ${ }^{37}$ However, there are always some drawbacks with these procedures such as long reaction time, ${ }^{26,33}$ high temperature, ${ }^{15}$ use of costly catalysts, ${ }^{23,24,35}$ high catalyst loading, ${ }^{22,31}$ use of an additional ultrasound ${ }^{19}$ or microwave oven ${ }^{30}$ etc. So, the development of new reagents with great efficiency, convenient procedure, and delivery of better yields is of great interest.

Recently, cobalt salts have been introduced as mild and extraordinarily efficient catalysts for various organic transformations. ${ }^{38}$ Particularly, cobalt(II) chloride is easily availability, inexpensive, water tolerant and operates under nearly neutral conditions, its further exploration for other 
organic transformations will be quite useful. As a part of our ongoing program in developing various new synthetic transformations using cheap and eco-friendly materials as catalysts, ${ }^{39}$ we herein wish to report our results on the synthesis of $\beta$-enaminones and $\beta$-enamino esters using a catalytic amount of $\mathrm{CoCl}_{2} \cdot 6 \mathrm{H}_{2} \mathrm{O}$ at room temperature under solvent-free condition (Scheme 1).<smiles>[R]C(=O)C([R])C([R])=O</smiles>

Scheme 1.

\section{Results and Discussion}

Our initial studies were focused on the optimization of the reaction conditions for the synthesis of $\beta$-enamino esters. Ethyl acetoacetate was chosen as a model substrate for the optimization process. The reaction of ethyl acetoacetate with aniline in the presence of $5 \mathrm{~mol} \%$ $\mathrm{CoCl}_{2} \cdot 6 \mathrm{H}_{2} \mathrm{O}$ at room temperature, without any solvent, afforded the target ethyl 3-(phenylamino)but-2-enoate (3e) in $95 \%$ yield. Lower catalyst loading can be used with only a marginal drop in reaction rate. With the optimized reaction condition, we next studied the reactions of a series of $\beta$-ketoesters with amines. The results presented in Table 1 indicate the generality of the method and efficacy of $\mathrm{CoCl}_{2} \cdot 6 \mathrm{H}_{2} \mathrm{O}$ as very mild catalyst. This method was successfully applied to enamination of simple linear $\beta$-ketoesters (a-j) and cyclic $\beta$-ketoesters $(\mathbf{k}-\mathbf{p})$. From the reaction of ethyl acetoacetate with various aryl amines, electronic effects were clearly observed. In general, aryl amines having no substituents or electron-donating substituents on the aromatic ring were more reactive, and afforded the corresponding products $\mathbf{3}$ in better yields (Table 1, 3e, 3g and 3h). An electron-withdrawing group had a strong deactivating effect, thus longer reaction time was required and the corresponding product was obtained in lower yield (Table 1, 2j). As expected, a steric interference was pronounced when the group is at the ortho-position of amine. In comparation to the synthesis of $\beta$-enamino esters $\mathbf{3 e - 3 h}$, a decrease of the yield for the synthesis of $\mathbf{3 i}$ was observed. It is noteworthy that optically active amine was converted into the corresponding $\beta$-enamino ester without any racemization or inversion by measuring its optical rotation and comparing with the literature value (Table 1, 3d).

This method is equally effective for symmetrical and unsymmetrical $\beta$-diketones. In the case of unsymmetrical $\beta$-diketones, the regiochemistry was controlled by the more reactive carbonyl group, which underwent preferential attack of amine. For example, 1-benzoylacetone reacted

Table 1. $\mathrm{CoCl}_{2} \cdot 6 \mathrm{H}_{2} \mathrm{O}$-catalyzed synthesis of $\beta$-enaminones and $\beta$-enamino esters

\begin{tabular}{|c|c|c|c|c|c|c|c|}
\hline Entry & $\mathrm{R}^{1}$ & & $\mathrm{R}^{2}$ & $\mathrm{R}^{3}$ & $\mathrm{R}^{4}$ & time / min & Yield / \% \\
\hline $\mathbf{a}$ & $\mathrm{Me}$ & & $\mathrm{H}$ & $\mathrm{OMe}$ & $\mathrm{CH}_{3}\left(\mathrm{CH}_{2}\right)_{3}$ & 15 & 95 \\
\hline b & $\mathrm{Me}$ & & $\mathrm{H}$ & OEt & $\mathrm{C}_{6} \mathrm{H}_{11}$ & 15 & 94 \\
\hline c & $\mathrm{Me}$ & & $\mathrm{H}$ & $\mathrm{OMe}$ & $\mathrm{H}_{2} \mathrm{C}=\mathrm{CHCH}_{2}$ & 15 & 95 \\
\hline d & $\mathrm{Me}$ & & $\mathrm{H}$ & $\mathrm{OMe}$ & $(R)-\mathrm{PhCH}\left(\mathrm{CH}_{3}\right)$ & 15 & 92 \\
\hline $\mathbf{e}$ & $\mathrm{Me}$ & & $\mathrm{H}$ & $\mathrm{OEt}$ & $\mathrm{Ph}$ & 50 & 95 \\
\hline f & $\mathrm{Me}$ & & $\mathrm{H}$ & $\mathrm{OMe}$ & $o-\mathrm{Me}-\mathrm{C}_{6} \mathrm{H}_{4}$ & 60 & 94 \\
\hline g & $\mathrm{Me}$ & & $\mathrm{H}$ & $\mathrm{OEt}$ & $p-\mathrm{Me}-\mathrm{C}_{6}^{6} \mathrm{H}_{4}^{4}$ & 45 & 93 \\
\hline h & $\mathrm{Me}$ & & $\mathrm{H}$ & OEt & $p$-OEt- $\mathrm{C}_{6} \mathrm{H}_{4}$ & 45 & 95 \\
\hline i & $\mathrm{Me}$ & & $\mathrm{H}$ & $\mathrm{OMe}$ & $2,6-\mathrm{Et}_{2}-\mathrm{C}_{6} \mathrm{H}_{3}$ & $7 \mathrm{~h}$ & 82 \\
\hline $\mathbf{j}$ & $\mathrm{Me}$ & & $\mathrm{H}$ & $\mathrm{OEt}$ & $p-\mathrm{Cl}-\mathrm{C}_{6} \mathrm{H}_{4}$ & $8 \mathrm{~h}$ & 75 \\
\hline k & $\mathrm{Me}$ & & & & $\mathrm{PhCH}_{2}$ & 30 & 91 \\
\hline 1 & $\mathrm{Me}$ & & & & $\mathrm{Ph}$ & 75 & 92 \\
\hline $\mathbf{m}$ & $\mathrm{Me}$ & & & & $p-\mathrm{OMe}-\mathrm{C}_{6} \mathrm{H}_{4}$ & 60 & 91 \\
\hline o & & $\left(\mathrm{CH}_{2}\right)_{3}$ & & & $\mathrm{Ph}$ & 90 & 93 \\
\hline $\mathbf{p}$ & & $\left(\mathrm{CH}_{2}\right)_{3}$ & & & $p$-OMe- $\mathrm{C}_{6} \mathrm{H}_{4}$ & 90 & 92 \\
\hline q & $\mathrm{Me}$ & & $\mathrm{H}$ & $\mathrm{Me}$ & $\mathrm{CH}_{3}\left(\mathrm{CH}_{2}\right)_{3}^{4}$ & 15 & 94 \\
\hline $\mathbf{r}$ & $\mathrm{Me}$ & & $\mathrm{H}$ & $\mathrm{Me}$ & $\mathrm{H}_{2} \mathrm{C}=\mathrm{CHCH}_{2}$ & 15 & 95 \\
\hline $\mathbf{s}$ & $\mathrm{Me}$ & & $\mathrm{H}$ & $\mathrm{Me}$ & $\mathrm{H}_{2} \mathrm{NCH}_{2} \mathrm{CH}_{2} \mathrm{CH}_{2}^{2}$ & 18 & $94^{\mathrm{b}}$ \\
\hline $\mathbf{t}$ & $\mathrm{Me}$ & & $\mathrm{H}$ & $\mathrm{Me}$ & $\mathrm{Ph}$ & 15 & 95 \\
\hline $\mathbf{u}$ & $\mathrm{Me}$ & & $\mathrm{H}$ & $\mathrm{Me}$ & $o-\mathrm{Me}-\mathrm{C}_{6} \mathrm{H}_{4}$ & 18 & 93 \\
\hline $\mathbf{v}$ & $\mathrm{Me}$ & & $\mathrm{H}$ & $\mathrm{Me}$ & $p-\mathrm{Me}-\mathrm{C}_{6}{ }_{6} \mathrm{H}_{4}$ & 12 & 95 \\
\hline $\mathbf{w}$ & $\mathrm{Me}$ & & $\mathrm{H}$ & $\mathrm{Me}$ & $o-\mathrm{Br}-\mathrm{C}_{6} \mathrm{H}_{4}$ & $8 \mathrm{~h}$ & 82 \\
\hline $\mathbf{x}$ & $\mathrm{Me}$ & & $\mathrm{H}$ & $\mathrm{Ph}$ & $\mathrm{Ph}$ & 100 & 75 \\
\hline $\mathbf{y}$ & $\mathrm{Me}$ & & $\mathrm{H}$ & $\mathrm{Ph}$ & $p$-OEt- $\mathrm{C}_{6} \mathrm{H}_{4}$ & 100 & 80 \\
\hline
\end{tabular}

${ }^{\mathrm{a}}$ Isolated yield. ${ }^{\mathrm{b}}$ The reaction was conducted with ecetylacetone $(10 \mathrm{mmol})$, propane-1,3-diamine $(5 \mathrm{mmol})$, and $\mathrm{CoCl}_{2} \cdot 6 \mathrm{H}_{2} \mathrm{O}(0.25 \mathrm{mmol})$. 
with aniline to obtain exclusively single regioisomer $\mathbf{3 x}$. When this reaction was conducted with symmetrical diamines, two equivalents of $\beta$-diketone were used to give the corresponding product with two enamino groups in high yields (Table 1, 3s). We also carried out the reaction of acetylacetone with equimolar amount of propane-1,3diamine, but cyclic product was not obtained.

The method was found that the chemoselectivity was also very good. Amine attacked only at the ketone carbonyl for both diketones and $\beta$-ketoesters. The $(Z)$-selectivity in the products derived from acylic diketones and $\beta$-ketoesters was secured by intramolecular hydrogen bonding. The ${ }^{1} \mathrm{H}$ NMR spectra analyses of products supported this sterostructure, in which the proton of the -NH- group appeared in the region of 8.6-10.2 ppm.

The reaction proceeds very cleanly without the formation of any by-products except water. Because the reaction can be performed using a solvent-free procedure, at the end of the reaction, the crude mixture can be directly charge on a chromatographic column to obtain the pure product, avoiding any tedious work up.

In summary, we have developed a new and efficient procedure for the preparation of $\beta$-enamino compounds catalysed by $\mathrm{CoCl}_{2} \cdot 6 \mathrm{H}_{2} \mathrm{O}$. This method offers several advantages such as (i) short reaction times; (ii) no excess of the reactants and catalyst is demanded; (iii) no solvent is employed; (iv) cheap and commercially available catalyst is applied; $(v)$ no work-up is need, since the crude mixture can be directly charged on a chromatography column for immediate purification, which leads to an useful and attractive process for the synthesis of this type of compounds simple by changing different substrates.

\section{Experimental}

Melting points were measured using a X-4 apparatus and are uncorrected. ${ }^{1} \mathrm{H}$ NMR spectra were taken with a Bruker 300 spectrometer in a $\mathrm{CDCl}_{3}$ solution with tetramethylsilane as an internal standard. IR spectra were obtained using Bruker-TENSOR 27 spectrometer instrument. Mass spectra were recorded on a GC-MS Thermofinnigan Polaris-Q mass spectrometer. The elemental analyses were carried out in an Elemental Vario EL analyzer.

General procedure for the synthesis of $\beta$-enamino compounds

A mixture of 1,3-dicarbonyl compounds (5 mmol), amines $(5 \mathrm{mmol})$ and $\mathrm{CoCl}_{2} \cdot 6 \mathrm{H}_{2} \mathrm{O}(0.25 \mathrm{mmol}, 58 \mathrm{mg})$ was stirred at room temperature for an appropriate time (see Table 1). After completion of the reaction, the crude products were separated by column chromatography on $\mathrm{Et}_{3} \mathrm{~N}$ pre-treated silica gel using petroleum ether (bp 60$\left.80{ }^{\circ} \mathrm{C}\right) /$ EtOAc $(10: 1)$ as eluent.

The spectral and analytical data of some representative compounds are given below.

\section{(Z)-Methyl 3-(allylamino)but-2-enoate $(3 \boldsymbol{c})^{40}$}

A yellowish oil; IR (neat) $v_{\max } / \mathrm{cm}^{-1}: 1655,1606 ;{ }^{1} \mathrm{H}$ $\operatorname{NMR}\left(\mathrm{CDCl}_{3}, 300 \mathrm{MHz}\right) \delta 1.91$ (s, 3H), 3.64 (s, 3H), 3.82$3.86(\mathrm{~m}, 2 \mathrm{H}), 4.99(\mathrm{~s}, 1 \mathrm{H}), 5.15-5.26(\mathrm{~m}, 2 \mathrm{H}), 5.83-5.94$ (m, 1H), 8.66 (br s, 1H, NH); EIMS m/z (\%): $155\left(\mathrm{M}^{+}, 39\right)$, 140 (26), 96 (100), 79 (29); Anal. Calc. for $\mathrm{C}_{8} \mathrm{H}_{13} \mathrm{NO}_{2}$ : C, $61.91 ; \mathrm{H}, 8.44 ; \mathrm{N}, 9.03$. Found: C, 62.12; H, 8.62; N, 8.88.

(Z)-methyl 3-((R)-1-phenylethylamino)but-2-enoate (3d)

A colorless liquid, $[\alpha]_{\mathrm{D}}^{20}:-550(c 1.02, \mathrm{EtOH})[-546]^{32}$; IR (neat) $v_{\text {max }} / \mathrm{cm}^{-1}: 1652,1607 ;{ }^{1} \mathrm{H}$ NMR $\left(\mathrm{CDCl}_{3}, 300 \mathrm{MHz}\right)$ $\delta 1.52$ (d, $J 6.9 \mathrm{~Hz}, 3 \mathrm{H}), 1.78$ (s, 3H), 3.68 (s, 3H), 4.49 (s, $1 \mathrm{H}), 4.64$ (q, $J 6.9 \mathrm{~Hz}, 1 \mathrm{H}), 7.22-7.38(\mathrm{~m}, 5 \mathrm{H}), 8.98$ (br s, 1H, NH); EIMS $m / z 219\left(\mathrm{M}^{+}, 41\right), 204$ (31), 172 (29), 145 (89), 105 (100), 84 (17); Anal. Calc. for $\mathrm{C}_{13} \mathrm{H}_{17} \mathrm{NO}_{2}$ : C, 71.21 ; H, 7.81; N, 6.39. Found: C, 61.48; H, 8.02; N, 6.18.

\section{(Z)-Methyl 3-(o-tolylamino)but-2-enoate (3f)}

A pale yellow solid, mp. $\left.28-30{ }^{\circ} \mathrm{C}\left[26-27{ }^{\circ} \mathrm{C}\right]\right]^{32}$; IR $(\mathrm{KBr}) v_{\max } / \mathrm{cm}^{-1}: 1648,1598 ;{ }^{1} \mathrm{H}$ NMR $\left(\mathrm{CDCl}_{3}, 300 \mathrm{MHz}\right)$ $\delta 1.85(\mathrm{~s}, 3 \mathrm{H}), 2.28(\mathrm{~s}, 3 \mathrm{H}), 3.68(\mathrm{~s}, 3 \mathrm{H}), 4.70(\mathrm{~s}, 1 \mathrm{H})$, 7.02-7.23 (m, 4H), 10.12 (br s, 1H, NH); EIMS m/z 205 $\left(\mathrm{M}^{+}, 44\right), 190$ (11)), 174 (16), 158 (14), 146 (30), 132 (100), 117 (21); Anal. Calc. for $\mathrm{C}_{12} \mathrm{H}_{15} \mathrm{NO}_{2}$ : C, 70.22; H, 7.37; N, 6.82. Found: C, 69.98; H, 7.52; N, 6.68.

\section{(Z)-Ethyl 3-(p-tolylamino)but-2-enoate $(3 g)^{41}$}

A yellow oil; IR (neat) $v_{\max } / \mathrm{cm}^{-1}: 1654,1608 ;{ }^{1} \mathrm{H}$ NMR $\left(\mathrm{CDCl}_{3}, 300 \mathrm{MHz}\right) \delta 1.29(\mathrm{t}, J 7.2 \mathrm{~Hz}, 3 \mathrm{H}), 1.96(\mathrm{~s}, 3 \mathrm{H})$, $2.33(\mathrm{~s}, 3 \mathrm{H}), 4.15(\mathrm{q}, J 7.2 \mathrm{~Hz}, 2 \mathrm{H}), 4.67(\mathrm{~s}, 1 \mathrm{H}), 6.98(\mathrm{~d}$, $J 8.4 \mathrm{~Hz}, 2 \mathrm{H}), 7.13(\mathrm{~d}, J 8.4 \mathrm{~Hz}, 2 \mathrm{H}), 10.28($ br s, $1 \mathrm{H}$, NH); EIMS m/z 219 (M+, 84), 14 (39), 146 (100), 132 (81), 91 (35); Anal. Calc. for $\mathrm{C}_{13} \mathrm{H}_{17} \mathrm{NO}_{2}$ : C, 71.21; H, 7.81; N, 6.39. Found: C, 71.46; H, 8.04; N, 6.16.

\section{(Z)-Ethyl 3-(p-ethoxyphenylamino)but-2-enoate (3h)}

A pale yellow solid, mp 55-56 $\left.{ }^{\circ} \mathrm{C}\left[53-54{ }^{\circ} \mathrm{C}\right]\right]^{32}$; IR (KBr) $v_{\max } / \mathrm{cm}^{-1}: 1652,1613 \mathrm{~cm}^{-1} ;{ }^{1} \mathrm{H}$ NMR $\left(\mathrm{CDCl}_{3}, 300\right.$ MHz) $\delta 1.29$ (t, J 7.2 Hz, 3H), 1.42 (t, J 7.2 Hz, 3H), 1.89 
(s, 3H), 4.02 (q, J $7.2 \mathrm{~Hz}, 2 \mathrm{H}), 4.15(\mathrm{q}, J 7.2 \mathrm{~Hz}, 2 \mathrm{H})$, $4.65(\mathrm{~s}, 1 \mathrm{H}), 6.85(\mathrm{~d}, J 8.7 \mathrm{~Hz}, 2 \mathrm{H}), 7.02(\mathrm{~d}, J 8.7 \mathrm{~Hz}$, 2H), 10.16 (br s, 1H, NH); EIMS m/z, 249 (M+1 $\left.\mathrm{M}^{+}, 17\right), 203$ (100), 174 (31), 147 (51), 118 (29), 91(9); Anal. Calc. for $\mathrm{C}_{14} \mathrm{H}_{19} \mathrm{NO}_{3}: \mathrm{C}, 67.45 ; \mathrm{H}, 7.68 ; \mathrm{N}$, 5.62. Found: $\mathrm{C}, 67.70$; $\mathrm{H}, 7.42 ; \mathrm{N}, 5.85$.

\section{(Z)-Methyl 3-(2,6-diethylphenylamino)but-2-enoate (3i)}

A pale yellow solid, mp $\left.27-28{ }^{\circ} \mathrm{C}\left[26-27{ }^{\circ} \mathrm{C}\right]\right]^{32}$; IR $(\mathrm{KBr}) \mathrm{V}_{\max } / \mathrm{cm}^{-1}: 1658,1607 ;{ }^{1} \mathrm{H} \mathrm{NMR}\left(\mathrm{CDCl}_{3}, 300 \mathrm{MHz}\right)$ $\delta 1.19(\mathrm{t}, J 7.8 \mathrm{~Hz}, 6 \mathrm{H}), 1.62(\mathrm{~s}, 3 \mathrm{H}), 2.52(\mathrm{q}, J 7.8 \mathrm{~Hz}$, $2 \mathrm{H}), 2.63(\mathrm{q}, J 7.8 \mathrm{~Hz}, 2 \mathrm{H}), 3.71(\mathrm{~s}, 3 \mathrm{H}), 4.87(\mathrm{~s}, 1 \mathrm{H})$, 7.13 (d, J 7.8 Hz, 2H), 7.19-7.24 (m, 1H), 9.88 (br s, $1 \mathrm{H}$, $\mathrm{NH})$; EIMS $m / z, 247\left(\mathrm{M}^{+}, 18\right), 174$ (100), 146 (33); Anal. Calc. for $\mathrm{C}_{15} \mathrm{H}_{21} \mathrm{NO}_{2}$ : C, 72.84; H, 8.65; N, 5.66. Found: C, 73.02; H, 8.46; N, 5.83 .

3-(1-(2-Methoxyphenylamino)ethylidene)-dihydrofuran$2(3 H)$-one $(3 \mathrm{~m})$

A pale yellow solid, mp $93-94{ }^{\circ} \mathrm{C}$; IR $(\mathrm{KBr}) \mathrm{v}_{\max } / \mathrm{cm}^{-1}$ : 1676, 1635; ${ }^{1} \mathrm{HNMR}\left(\mathrm{CDCl}_{3}, 300 \mathrm{MHz}\right) \delta 1.90(\mathrm{~s}, 3 \mathrm{H})$, $2.88(\mathrm{t}, J 7.8 \mathrm{~Hz}, 2 \mathrm{H}), 3.78(\mathrm{~s}, 3 \mathrm{H}), 4.34(\mathrm{t}, J 7.8 \mathrm{~Hz}, 2 \mathrm{H})$, $6.85(\mathrm{~d}, J 7.8 \mathrm{~Hz}, 1 \mathrm{H}), 6.98(\mathrm{~d}, J 7.8 \mathrm{~Hz}, 1 \mathrm{H}), 9.76$ (br s, $1 \mathrm{H}, \mathrm{NH})$; Anal. Calc. for $\mathrm{C}_{13} \mathrm{H}_{15} \mathrm{NO}_{3}: \mathrm{C}, 66.94 ; \mathrm{H}, 6.48$; N, 6.00. Found: C, 66.79; H, 6.25; N, 6.20.

\section{1,3-Bis-(2-acetyl-1-methylvinylamino)propane (3s)}

A dark brown solid, mp $50-52{ }^{\circ} \mathrm{C}\left[51{ }^{\circ} \mathrm{C}\right]^{42}$; IR (KBr) $v_{\max } / \mathrm{cm}^{-1}: 1608,1570 ;{ }^{1} \mathrm{H}$ NMR $\left(\mathrm{CDCl}_{3}, 300 \mathrm{MHz}\right) \delta 1.89$ $(\mathrm{t}, J 6.3 \mathrm{~Hz}, 2 \mathrm{H}), 1.92$ (s, 6H), 2.01 (s, 6H), 3.38 (q, J 6.3 $\mathrm{Hz}, 4 \mathrm{H}$ ), 5.00 (s, 2H), 10.92 (br s, 1H, NH); Anal. Calc. For $\mathrm{C}_{13} \mathrm{H}_{22} \mathrm{~N}_{2} \mathrm{O}_{2}$ : C, 65.51; $\mathrm{H}, 9.30 ; \mathrm{N}, 11.75$. Found: $\mathrm{C}$, 65.69; H, 9.18; N, 11.85 .

\section{(Z)-4-(o-Tolylamino)pent-3-en-2-one (3u)}

A pale yellow solid, mp $38-40{ }^{\circ} \mathrm{C}\left[37-38{ }^{\circ} \mathrm{C}\right]^{32}$; IR (KBr) $v_{\text {max }} / \mathrm{cm}^{-1}: 1595,1560 ;{ }^{1} \mathrm{HNMR}\left(\mathrm{CDCl}_{3}, 300 \mathrm{MHz}\right)$ $\delta 1.88(\mathrm{~s}, 3 \mathrm{H}), 2.12(\mathrm{~s}, 3 \mathrm{H}), 2.29(\mathrm{~s}, 3 \mathrm{H}), 5.21(\mathrm{~s}, 1 \mathrm{H})$, 7.07-7.25 (m, 4H), 12.36 (br s, 1H, NH); EIMS m/z. 189 $\left(\mathrm{M}^{+}, 44\right), 174$ (100), 146 (63), 131 (44), 91 (11); Anal. Calc. for $\mathrm{C}_{12} \mathrm{H}_{15} \mathrm{NO}$ : C, 76.16; H, 7.99; N, 7.40. Found: C, 76.02; H, 8.25; N, 7.65.

\section{(Z)-3-(4-Ethoxy-phenylamino)-1-phenyl-but-2-en-1-one (3y)}

A yellow solid, mp 84-85 ${ }^{\circ} \mathrm{C}$; IR (KBr) $v_{\max } / \mathrm{cm}^{-1}: 1599$, 1504; ${ }^{1} \mathrm{H} \mathrm{NMR}\left(\mathrm{CDCl}_{3}, 300 \mathrm{MHz}\right) \delta 1.40(\mathrm{t}, J 6.9 \mathrm{~Hz}$,
3H), 2.05 (s, 3H), 4.02 (q, J $6.9 \mathrm{~Hz}, 2 \mathrm{H}), 5.85(\mathrm{~s}, 1 \mathrm{H})$, $6.89(\mathrm{~d}, J 9.0 \mathrm{~Hz}, 2 \mathrm{H}), 7.10(\mathrm{~d}, J 8.7 \mathrm{~Hz}, 2 \mathrm{H}), 7.44-7.47$ (m, 3H), 7.90-7.94 (m, 2H), 12.88 (br s, 1H, NH); Anal. Calc. for $\mathrm{C}_{18} \mathrm{H}_{19} \mathrm{NO}_{2}: \mathrm{C}, 76.84 ; \mathrm{H}, 6.81 ; \mathrm{N}, 4.98$. Found: C, 76.95; H, 6.76; N, 4.72 .

\section{Acknowledgments}

We thank the Science Research Foundation of Hebei Normal University, Natural Science Foundation of Hebei Province (B2005000151) and the Natural Science Foundation of Hebei Education Department (2006318) for financial support.

\section{References}

1. Caprathe, B. W.; Jaen, J. C.; Wise, L. D.; Heffner, T. G.; Pudsley, T. A.; Melther, L. T.; Parvez, M.; J. Med. Chem. 1991, 34, 3726.

2. Gatta, F.; Del Giudice, M. R.; Pomponi, M.; Marta, M.; Heterocycles 1992, 34, 991.

3. Scott, K. R.; Edafiogho, I. O.; Richardson, E. C.; Farrar, V. A.; Moore, J. A.; Tietz, E. I.; Hinko, C. N.; Chang, H.; El-Assadi, A.; Nicholson, J. M.; J. Med. Chem. 1993, 36, 1947.

4. Palmieri, G.; Cimarelli, C.; J. Org. Chem. 1996, 61, 5557.

5. Bartoli, G.; Cimarelli, C. Marcantoni, E.; Palmieri, G.; Petrini, M.; J. Org. Chem. 1994, 59, 5328; Cimarelli, C.; Giuli, S.; Palmieri, G.; Eur. J. Org. Chem. 2006, 1017; Harris, M. I. N. C.; Braga, A. C. H.; J. Braz. Chem. Soc. 2004, 15, 971; Zanatta, N.; Squizani, A. M. C.; Fantinel, L.; Nachtigall, F. M.; Borchhardt, D. M.; Bonacorso, H. G.; Martins, M. A. P.; J. Braz. Chem. Soc. 2005, 16, 1255.

6. Beholz, L. G.; Benovsky, P.; Ward, D. L.; Bata, N. S.; Stille, J. R. J. Org. Chem. 1997, 62, 1033.

7. Kascheres, C. M.; J. Braz. Chem. Soc. 2003, 14, 945; Elassar, A.-Z. A.; El-Khair, A. A.; Tetrahedron 2003, 59, 8463; Ferraz, H. M. C.; Pereira, F. L. C.; Quim. Nova 2004, $27,89$.

8. Kosugi, M.; Koshiba, M.; Atoh, A.; Sano, H.; Migita, T.; Bull. Chem. Soc. Jpn. 1986, 59, 677.

9. Sakamoto, T.; Nagano, T.; Kondo, Y.; Yamanaka, H.; Synthesis 1990, 215.

10. Katritzky, A. R.; Fang, Y.; Donkor, A.; Xu, J.; Synthesis 2000, 2029; Katritzky, A. R.; Hayden, A. E.; Kirichenko, K.; Pelphrey, P.; Ji, Y.; J. Org. Chem. 2004, 69, 5108.

11. Bartoli, G.; Cimarelli, C.; Dalpozzo, R.; Palmieri, G.; Tetrahedron 1995, 51, 8613.

12. Hannick, S. M.; Kishi, Y.; J. Org. Chem. 1983, 48, 3833.

13. Seko, S.; Tani, N.; Tetrahedron Lett. 1998, 39, 8117.

14. Reddy, D. S.; Rajale, T. V.; Shivakumar, R. K.; Iqbal, J.; Tetrahedron Lett. 2005, 46, 979.

15. Singh, R. V.; Tandon, J. P.; Journal Fur Praktische Chemie 1979, 321, 151. 
16. Sano, T.; Horiguchi, Y.; Toda, J.; Imafuku, K.; Tsuda, Y.; Chem. Pharm. Bull. 1984, 32, 497.

17. Aminabhavi,T. M.; Biradar, N. S.; Patil, S. B.; Indian J. Chem. 1986, 25A, 285.

18. Bieräugel, H.; Akkerman, J. M.; Armande, J. C. L.; Pandit, U. K.; Recueil Des Travaux Chimiques Des Pays-BasJournal Of The Royal Netherlands Chemical Society 1976, 95, 266.

19. Brandt, C. A.; da Silva, A. C. M. P.; Pancote, C. G.; Brito, C. L.; da Silveira, M. A. B.; Synthesis 2004, 1557.

20. Itefane, B.; Polanc, S.; Synlett 2004, 698.

21. Bartoli, G.; Bosco, M.; Locatelli, M.; Marcantoni, E. Melchiorre, P.; Sambri, L.; Synlett 2004, 239.

22. Khodaei, M. M.; Khosropour, A. R.; Kookhazadeh, M.; Synlett 2004, 1980.

23. Arcadi, A.; Bianchi, G.; Di Giuseppe, S.; Marinelli, F.; Green Chem. 2003, 5, 64.

24. Khodaei, M. M.; Khosropour, R.; Kookhazadeh, M.; Can. J. Chem. 2005, 83, 209; Khosropour, A. R.; Khodaei, M. M.; Kookhazadeh, M.; Tetrahedron Lett. 2004, 45, 1725.

25. Silva, F. C.; De Souza, M. C. B. V.; Ferreira, V. F.; Sabino, S. J.; Antunes, O. A. C.; Catal. Commun. 2004, 5, 151.

26. Gao, Y.-H.; Zhang, Q.-H.; Xu, J.-X.; Synth. Commun. 2004, 34, 909.

27. Texier-Boullet, F.; Klein, B.; Hamelin, J.; Synthesis 1986, 409; Braibante, M. E. F.; Braibante, H. S.; Missio, L.; Andricopulo, A.; Synthesis 1994, 898.

28. Zhang, Z.-H.; Song, L.-M.; J. Chem. Res. 2005, 817.

29. Gholap, A. R.; Chakor, N. S.; Daniel, T.; Lahoti, R. J.; Srinivasan, K. V.; J. Mol. Catal. A: Chem. 2006, 245, 37.

30. Braibante, H. T. S; Braibante, M. E. F.; Rosso, G. B.; Oriques, D. A.; J. Braz. Chem. Soc. 2003, 14, 994; Braibante, M. E. F.; Braibante, H. T. S.; Morel, A. F.; Costa, C. C.; Lima, M. G.; J. Braz. Chem. Soc. 2006, 17, 184.

31. Gogoi, S.; Bhuyan, R.; Barua, N. C.; Synth. Commun. 2005, 35, 2811.

32. Zhang, Z.-H.; Yin, L.; Wang, Y.-M.; Adv. Synth. Catal. 2006, 348, 184.

33. Vohra, R. K.; Renaud, J.-L.; Bruneau, C.; Collect. Czech. Chem. Commun. 2005, 70, 1943.
34. Bhosale, R. S.; Suryawanshi, P. A.; Ingle, S. A.; Lokhande, M. N.; More, S. P.; Mane, S. B.; Bhosale, S. V.; Pawar, R. P.; Synlett 2006, 933.

35. Yadav, J. S.; Kumar, V. N.; Rao, R. S.; Priyadarshini, A. D.; Rao, P. P.; Reddy, B. V. S.; Nagaiah, K.; J. Mol. Catal. A: Chem. 2006, 256, 234.

36. Das, B.; Venkateswarlu, K.; Majhi, A.; Reddy, M. R.; Reddy, K. N.; Rao, Y. K.; Ravikumar, K.; Sridhar, B.; J. Mol. Catal. A: Chem. 2006, 246, 276.

37. Stefani, A. A.; Costa. I. M.; de O. Silva, D.; Synthesis 2000 , 1526.

38. Ohmiya, H.; Tsuji, T.; Yorimitsu, H. Oshima, K.; Chem. Eur. J. 2004, 10, 5640; Korn, T. J.; Knochel, P.; Angew. Chem., Int. Ed. 2005, 44, 2947; Amatore, M.; Gosmini, C.; Perichon, J.; Eur. J. Org. Chem. 2005, 989; Ikeda, Y.; Yorimitsu, H.; Shinokubo, H.; Oshim, K.; Adv. Synth. Catal. 2004, 346, 1631; Gao, G.-Y.; Harden, J. D.; Zhang, X. P. Org. Lett. 2005, 7, 3191; Waser, J.; Nambu, H.; Carreira, E. M. J. Am. Chem. Soc. 2005, 127, 8294; De, S. K. Tetrahedron Lett. 2004, 45, 1035; Velusamy, S.; Borpuzari, S.; Punniyamurthy, T. Tetrahedron 2005, 61, 2011; Velusamy. S.; Punniyamurthy, T. Tetrahedron Lett. 2004, 45, 4917; Velusamy. S.; Kumar, J. S. K.; Punniyamurthy, T. Tetrahedron Lett. 2004, 45 , 203; Sundararajan, G.; Vijayakrishna, K.; Varghese, B. Tetrahedron Lett. 2004, 45, 8253; Chang, H.-T.; Jeganmohan, M.; Cheng, C.H. Chem. Commun. 2005, 4955; Kuno, A.; Saino, N.; Kamachi, T. Okamoto, S. Tetrahedron Lett. 2006, 47, 2591; Korn, T. J.; Schade, M. A.; Wirth, S.; Knochel, P. Org. Lett. 2006, 8, 725; Paraskar, A. S.; Sudalai, A.; Tetrahedron 2006, 62, 4907.

39. Zhang, Z.-H.; J. Chem. Res., Synop. 2004, 753; Zhang, Z.-H.; Monatsh. Chem. 2005, 136, 1191; Mo, L.-P.; Ma, Z.-C.; Zhang, Z.-H.; Synth. Commun. 2005, 35, 1997; Zhang, Z.-H.; Yang, S.-T.; Lin, J.; Synth. Commun. 2006, 36, 1645; Cui, Z.-S.; Zhang, Z.-H.; Liu, S.-F.; J. Chem. Res. 2006, 390; Zhang, Z.H.; Lin, J.; Synth. Commun. 2006, 36, in press.

40. Morita, I.; Haruta, Y.; Tomita, T.; Tsuda, M.; Kandori, K.; Kise, M.; Kimura, K.; Chem. Pharm. Bull. 1987, 35, 4819.

41. Potšil, T.; J. Chromatogr. 1984, 312, 387.

42. McDougall, R. H.; Malik, S. H.; J. Chem. Soc., C 1969, 2044.

Received: June 22, 2006

Published on the web: November 7, 2006 